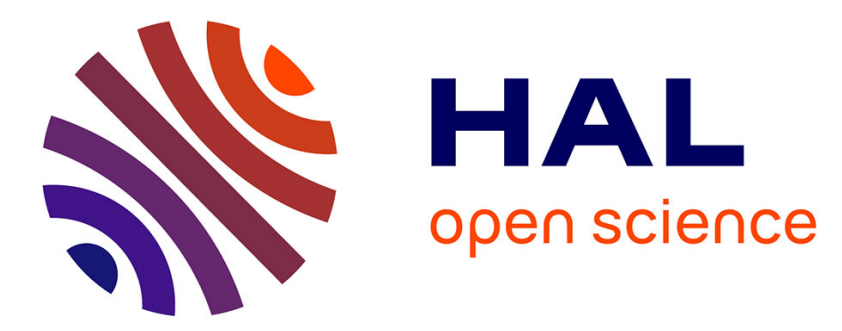

\title{
Polarization and configuration dependence of beam self-focusing in photorefractive LiNbO3
}

J. Safioui, M. Chauvet, F. Devaux, V. Coda, F. Pettazzi, M. Alonzo, E. Fazio

\section{To cite this version:}

J. Safioui, M. Chauvet, F. Devaux, V. Coda, F. Pettazzi, et al.. Polarization and configuration dependence of beam self-focusing in photorefractive LiNbO3. Journal of the Optical Society of America B, 2009, 26 (3), pp.487-492. 10.1364/JOSAB.26.000487 . hal-00441844

\section{HAL Id: hal-00441844 \\ https://hal.science/hal-00441844}

Submitted on 4 May 2021

HAL is a multi-disciplinary open access archive for the deposit and dissemination of scientific research documents, whether they are published or not. The documents may come from teaching and research institutions in France or abroad, or from public or private research centers.
L'archive ouverte pluridisciplinaire HAL, est destinée au dépôt et à la diffusion de documents scientifiques de niveau recherche, publiés ou non, émanant des établissements d'enseignement et de recherche français ou étrangers, des laboratoires publics ou privés. 


\title{
Polarization and configuration dependence of beam self-focusing in photorefractive $\mathrm{LiNbO}_{3}$
}

\author{
J. Safioui, ${ }^{1, *}$ M. Chauvet, ${ }^{1}$ F. Devaux, ${ }^{1}$ V. Coda, ${ }^{2}$ F. Pettazzi, ${ }^{3}$ M. Alonzo, ${ }^{3}$ and E. Fazio ${ }^{3}$ \\ ${ }^{1}$ Département d'Optique, Institut FEMTO-ST, UMR CNRS 6174, Université de Franche-Comté 16, route de Gray, \\ 25030 Besançon cedex, France \\ ${ }^{2}$ Laboratoire Matériaux Optiques, Photonique et Systèmes, UMR CNRS 7132, Université Paul-Verlaine-Metz- \\ Supélec, 2 rue Edouard Belin, 57070 Metz, France \\ ${ }^{3}$ Dipartimento di Energetica, Università La Sapienza Via Antonio Scarpa 16, I-00161 Roma, Italy \\ *Corresponding author: jassem.safioui@univ-fcomte.fr
}

\begin{abstract}
We present numerical and experimental analyses showing the formation of $(2+1) \mathrm{D}$ spatial photorefractive solitons at visible wavelengths in electrically biased lithium niobate crystals for ordinary and extraordinary light polarizations. Similarly sized self-trapped beams are observed for both polarizations, despite the polarizationdependent electro-optic coefficients. The tensorial character of the photovoltaic effect is shown to play a key role. The soliton-induced waveguides are able to properly guide telecommunication wavelengths. Finally, a higher degree of anisotropy is observed for ordinary polarized solitons for specific electro-optic configurations, which reveals the presence of the photorefractive field component perpendicular to the applied field. Experimental results are confirmed by a time-dependent numerical model.
\end{abstract}

\section{INTRODUCTION}

Since the first observation of optical spatial solitons by Kerr in the late 1980s [1], spatial solitons have been studied in various nonlinear media [2-21]. These self-trapped beams that propagate without alteration of their transverse profiles have been observed, for instance, in quadratic media [13] and also at very low power intensity in liquid crystals $[7,14,15]$ or photorefractive media [2-4,6,10-12,16], with recent studies devoted to propagation in periodic nonlinear media [5,17]. Optical nonlinear effects are essentially light polarization sensitive, which inspires vectorial analysis. Some media, such as Kerr AlGaAs [18,19], show weak soliton polarization dependence while others, like liquid crystal $[20,21]$ or photorefractive (PR) materials, possess features that enhance anisotropy. In particular, the electro-optic effect, which is central in liquid crystals and PR media, explains light polarization sensitivity. However, polarization dependence of a scalar spatial soliton formation has been analyzed primarily in a one-dimensional (1D) case $(1+1) \mathrm{D}$ [22,23]. For photovoltaic screening solitons at the heart of this work, not only the electro-optic effect but also the photovoltaic effect is a function of light polarization. This motivates the present study where the case of a photovoltaic screening soliton in a $\mathrm{LiNbO}_{3}$ crystal is treated in the frame of a $(2+1) \mathrm{D}$ model. In addition, the space-charge field component perpendicular to the crystal $c$ axis, which is usually neglected, is shown to affect soliton formation for particular configurations.

Lithium niobate $\left(\mathrm{LiNbO}_{3}\right)$, which is largely available and frequently used in the photonic and optoelectronics industry, is suitable for the formation of photorefractive spatial solitons. Dark photovoltaic solitons were first ob- served using defocusing nonlinearity [24,25], while bright photovoltaic solitons were observed in a more exotic ferroelectric crystal [26]. More recently, bright solitons have been demonstrated in $\mathrm{LiNbO}_{3}$ using an external applied field opposite and higher than the photovoltaic field [10]. Bright solitons are easy to form and give rise to memorized circular low-loss waveguides inside a bulk medium. Bright spatial solitons can thus be considered as a simple and low-cost technique to form tridimensional optical circuits in $\mathrm{LiNbO}_{3}$ that compare favorably with other techniques such as direct UV writing [27]. Many applications could be envisioned, such as compact optical information processing systems [28,29].

Beam self-focusing in $\mathrm{LiNbO}_{3}$ has been exclusively studied for extraordinary polarization that benefits from the strong electro-optic coefficient $r_{33}$. In this work we present the possibility of using ordinary polarization to create bright spatial solitons and efficient circular waveguides in $\mathrm{LiNbO}_{3}$. The experimental results are explained throughout a time-dependent $3 \mathrm{D}$ numerical model. We show that most efficient waveguides are induced with ordinary polarized solitons when probed by extraordinary light, as confirmed by guiding tests at telecommunication wavelengths. Additionally, the presence of the space-charge field component perpendicular to the applied field, which is usually neglected for PR solitons, is shown to have an impact on beams for particular configurations.

\section{THEORETICAL BACKGROUND AND NUMERICAL MODEL}

The system of Eqs. (1), suitable to describe the photorefractive effect in a medium with one deep-level donor and 
where transport is electron-dominated, is used to model beam self-trapping in $\mathrm{LiNbO}_{3}$ :

$$
\begin{aligned}
\frac{\partial N_{D}^{+}}{\partial t} & =s\left(I+I_{d}\right)\left(N_{D}-N_{D}^{+}\right)-\gamma n_{e} N_{D}^{+}, \\
\rho & =e\left(N_{D}^{+}-N_{A}-n_{e}\right), \\
\vec{J} & =e \mu n_{e} \vec{E}+\mu k_{\mathrm{B}} T \vec{\nabla} n_{e}+\beta_{p h}\left(N_{D}-N_{D}^{+}\right) I \vec{c}, \\
\frac{\partial \rho}{\partial t} & =-\vec{\nabla} \cdot \vec{J}, \\
\vec{E}(\vec{r}) & =\frac{1}{4 \pi[\varepsilon]} \iiint_{v} \rho\left(\vec{r}^{\prime}\right) \frac{\vec{r}-\vec{r}^{\prime}}{\left|\vec{r}-\vec{r}^{\prime}\right|^{3}} \mathrm{~d} V,
\end{aligned}
$$

where $I$ is the light intensity; $I_{d}$ is the equivalent dark intensity; $N_{A}, N_{D}, N_{D}^{+}$, and $n_{e}$ are the densities of shallow acceptors, deep donors, deep ionized donors, and free electrons, respectively; $s$ is the photo-excitation coefficient; $\gamma$ is the recombination constant; $k_{\mathrm{B}}$ is the Boltzmann constant; $\mu$ is the electron mobility; and $T$ is the crystal temperature. We assume a photovoltaic effect throughout $\beta_{p h}$, which depends on polarization. $\beta_{p h}=\beta_{p h 31}$ and $\beta_{p h}=\beta_{p h 33}$ for ordinary and extraordinary beam polarization in a $\mathrm{LiNbO}_{3}$ crystal, respectively. [ $\left.\varepsilon\right]$ is the static dielectric tensor, $\rho$ is the charge density, and $\vec{J}$ is the current density. Note that in this model the space-charge field $\vec{E}(\vec{r})$ produced in a point location by the charge density $\rho$ is expressed through the integral Eq. (1e) ( $\mathrm{d} V$ being an elementary volume), which is easier to solve by a 3D discrete fast Fourier transform than the usual Poisson equation. To obtain the evolution of the light-induced space-charge field $\vec{E}$, a numerical resolution of the system is necessary [30].

From the set of Eqs. (1), usual assumptions are made. The density of acceptors is assumed to be greater than the density of free electrons $\left(N_{A} \gg n_{e}\right)$. Moreover, the generation time of free electrons is neglected as compared with the characteristic evolution time of the space charge. Following these hypotheses we obtain from Eqs. (1a)-(1d),

$$
\begin{aligned}
\tilde{n}_{e}= & \frac{\xi\left(I+I_{d}\right)\left(\tilde{N}_{D}-\tilde{N}_{D}^{+}\right)}{\tilde{N}_{D}^{+}}, \\
\frac{\partial \widetilde{\rho}}{\partial t}= & -\mu E_{0}\left\{\vec{\nabla}\left[\tilde{n}_{e}\right] \cdot \overrightarrow{\widetilde{E}}+\tilde{n}_{e} \vec{\nabla} \cdot \overrightarrow{\widetilde{E}}+\frac{k_{B} T}{e E_{0}} \Delta \tilde{n}_{e}\right\} \\
& -\mu \xi E_{p h}\left(\tilde{N}_{D}-\tilde{N}_{D}^{+}\right) I \vec{c},
\end{aligned}
$$

where $\tilde{n}_{e}, \tilde{N}_{D}$, and $\tilde{N}_{D}^{+}$are free electrons, donors, and ionized donor densities normalized to $N_{A} ; \widetilde{\rho}$ is the spacecharge density normalized to $e N_{A}, \xi=s / \gamma N_{A}$; and $E_{p h}$ is the photovoltaic field given by

$$
E_{p h}=\frac{\beta_{p h} \gamma N_{A}}{e \mu s} .
$$

For a monochromatic beam of wavelength $\lambda$, light propagation is given by the paraxial wave equation (PWE)

$$
\frac{\partial}{\partial z} A(x, y, z)=i \frac{1}{2 k} \nabla_{\perp}^{2} A(x, y, z)+i \frac{2 \pi}{\lambda} \Delta n A(x, y, z),
$$

where $\nabla_{\perp}^{2}=\left(\partial^{2} / \partial x^{2}, \partial^{2} / \partial y^{2}\right), \mathrm{A}_{\mathrm{x}}$ and $\mathrm{A}_{\mathrm{y}}$ are the transverse components of the optical field with $\mathrm{I},=|\mathrm{A}|^{2}$, and $\mathrm{k}$ $=2 \pi \mathrm{n} / \lambda$ is the wave vector in the unperturbed medium of refractive index $n$. Note that the PWE is not strictly valid for an extraordinary polarized beam because of the crystal anisotropy as shown in [31]. However, experimental results presented farther on in the paper are predicted adequately with Eq. (5), which justifies that model refinement is not necessary.

The index perturbation $\Delta n$ induced by the photorefractive space-charge field by electro-optic effect is given by

$$
\Delta n \simeq-\frac{1}{2} n^{3}[r]\left[\begin{array}{c}
E_{X} \\
E_{Y} \\
E_{Z}
\end{array}\right],
$$

with $[r]$ as the linear electro-optic tensor of $\mathrm{LiNbO}_{3}$ and $\left(\mathrm{E}_{\mathrm{X}}, \mathrm{E}_{\mathrm{Y}}, \mathrm{E}_{\mathrm{Z}}\right)$ as the space-charge field components.

For a given crystallographic configuration and light polarization, the numerical method consists of solving Eq. (1e) by 3D discrete fast Fourier transform (2) and (3) by the iterative method to obtain the space-charge field at a given time step, and the refractive index perturbation is deduced from Eq. (6) [30]. Light propagation is simultaneously calculated in each successive elementary propagation step by the split-step Fourier method. This process can be repeated until a steady-state regime is reached.

In this work we consider a standard configuration where an external field is applied along the $\mathrm{LiNbO}_{3} z$ axis while a beam propagates perpendicular to the $z$ axis. Note that the $z$ axis corresponds to the $\mathrm{LiNbO}_{3} c$ crystalline axis.

We first present the case of a $532 \mathrm{~nm}$ beam, either extraordinary or ordinary, polarized propagating along the $y$ crystallographic axis. Considering a null electric field component along propagation $\left(\mathrm{E}_{\mathrm{Y}}=0\right)$, index perturbations induced by the electro-optic effect [32] for extraordinary and ordinary polarization are given by Eqs. (7) and (8) for this specific configuration, respectively:

$$
\begin{aligned}
& \Delta n_{Z} \simeq-\frac{1}{2} n_{e}^{3} r_{33} E_{Z}, \\
& \Delta n_{X} \simeq-\frac{1}{2} n_{o}^{3} r_{13} E_{Z} .
\end{aligned}
$$

With $\mathrm{r}_{33}=32 \mathrm{pm} / \mathrm{V}$ and $\mathrm{r}_{13}=9,4 \mathrm{pm} / \mathrm{V}$, note that the $E_{X}$ component of the space-charge field does not play a role in the index change.

Parameters of the numerical simulation are optical power $114 \mu \mathrm{W}$, applied field $40 \mathrm{kV} / \mathrm{cm}, \mathrm{N}_{A}=2$ $\times 10^{26} \mathrm{~cm}^{-3} \mathrm{~N}_{D} / \mathrm{N}_{A}=2$, and $\mathrm{I}_{d}=\mathrm{I}_{\max } / 100$. A $12 \mu \mathrm{m}$ beam 
FWHM is considered at the input face of a $20 \mathrm{~mm}$ long crystal. Other parameters are taken from [30].

For an extraordinary polarized beam, calculated intensity distribution along with index perturbation and transverse space-charge field components at the crystal output are presented in Fig. 1 for a photovoltaic field $E_{p h}$ set to $-35 \mathrm{kV} / \mathrm{cm}$. This value of $E_{p h}$ is chosen to obtain a selftrapped beam when the best confinement is reached. Images correspond to an illumination time leading to the best confinement. A slightly elliptical spot (Fig. 1(a)) is obtained $\left(\mathrm{FWHM}_{z}=10 \mu \mathrm{m}, \mathrm{FWHM}_{x}=14 \mu \mathrm{m}\right)$, which is consistent with the asymmetric index distribution (Fig. 1(b)) produced by the transverse component $E_{Z}$ of the spacecharge field (Fig. 1(c)). Such a distribution is in accordance with previously published results on a $2 \mathrm{D}$ photorefractive model [33]. Component $E_{X}$ of the space-charge field, which is perpendicular to the applied field, has an antisymmetric distribution with four lobes as shown in (Fig. 1(d)). This component does not influence the refractive index distribution for the present electro-optic configuration but, however, contributes to charge displacement.

For an ordinary polarized beam, calculated intensity distribution, along with index perturbation and transverse space-charge field components at the crystal output, are presented in Fig. 2. $E_{p h}$ is arbitrarily taken identically as the one for extraordinary polarization, despite the fact that it could be polarization-dependent throughout the photovoltaic coefficient. In such a case we can observe that the trapped beam $\mathrm{FWHM}_{x}=38 \mu \mathrm{m}, \mathrm{FWHM}_{z}$ $=24 \mu \mathrm{m}$ is less confined compared with extraordinary polarized light due to the lower value of the electro-optic coefficient $r_{13}$ associated with ordinary polarization, which is approximately three times lower than the $r_{33}$ associated with extraordinary polarization.

For different configurations, the space-charge field component perpendicular to the applied field can also affect the self-focusing behavior. As an example, we still consider a $\mathrm{LiNbO}_{3}$ crystal biased along the $z$ axis but not for a beam propagating along the $x$ crystallographic axis. For this particular configuration and with ordinary polarization, both $E_{Y}$ and $E_{Z}$ components contribute to the in-
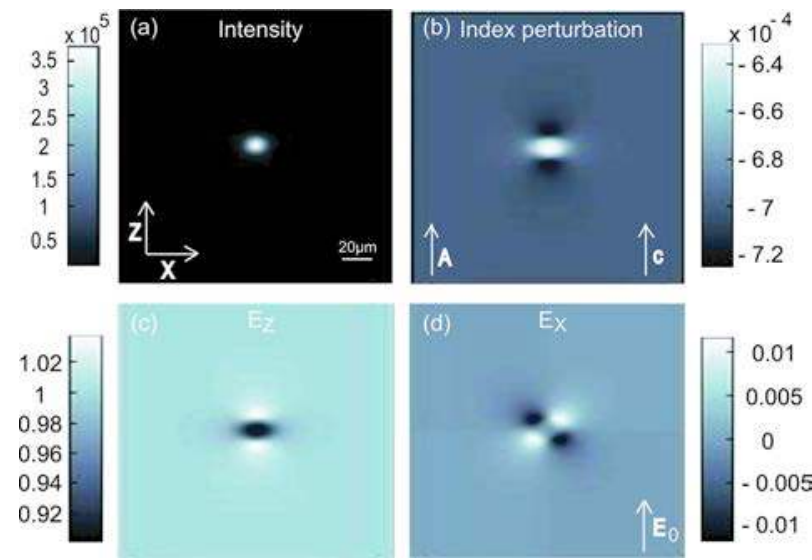

Fig. 1. (Color online) Calculated light intensity distribution for (a) extraordinary polarization, refractive index distribution and (b) transverse components (c) $E_{Z}$ and (d) $E_{X}$ of the space-charge field normalized to $E_{0}$ at the exit face for light propagation along the $y$ crystallographic axis in a $20 \mathrm{~mm}$ long $\mathrm{LiNbO}_{3}$ crystal.
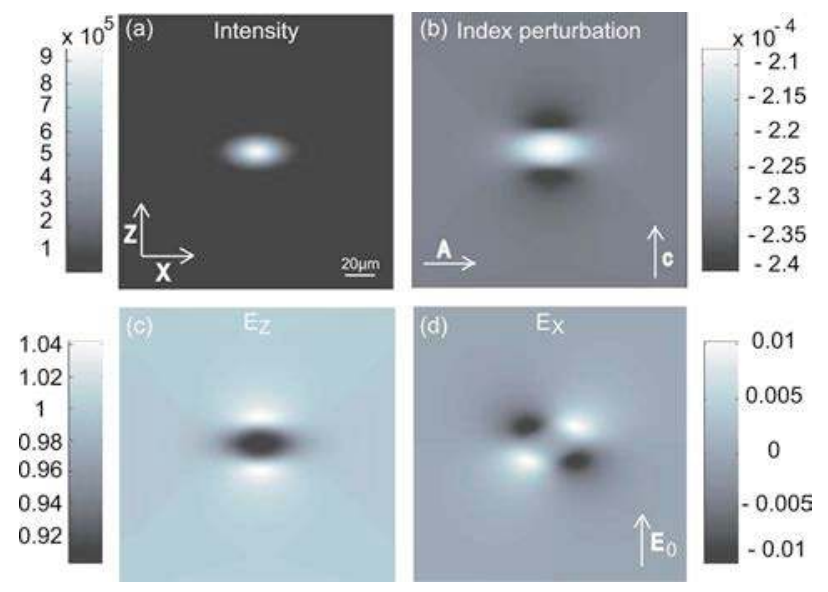

Fig. 2. (Color online) Calculated light intensity distribution for (a) ordinary polarization, (b) refractive index distribution, and transverse components (c) $E_{Z}$ and (d) $E_{X}$ of the space-charge field normalized to $E_{0}$ at the exit face for light propagation along the $y$ crystallographic axis in a $20 \mathrm{~mm}$ long $\mathrm{LiNbO}_{3}$ crystal.

dex change throughout the two electro-optic coefficients $\mathrm{r}_{13}$ and $\mathrm{r}_{22}=6,7 \mathrm{pm} / \mathrm{V}$, according to the expression

$$
\Delta n_{Y} \simeq-\frac{1}{2} n_{o}^{3}\left(r_{22} E_{Y}+r_{13} E_{Z}\right) .
$$

In this case, numerical simulations reveal a higher degree of anisotropy as shown in Fig. 3. An obvious consequence is the formation of an output-trapped beam with a tilted elliptical intensity distribution (Fig. 3(a)). This tilt is due to the asymmetric shape of $E_{Y}$ (Fig. 3(d)) that, added to $E_{Z}$, gives rise to the rotated refractive index perturbation (Fig. 3(b)). This characteristic is easier to visualize during the initial self-focusing of wide beams since weak variations of the refractive index have a strong influence on light distribution. On the contrary, beam tilt is hardly noticeable for strongly confined self-trapped beams since the amplitude of $E_{Z}$ prevails over the $E_{Y}$ component.
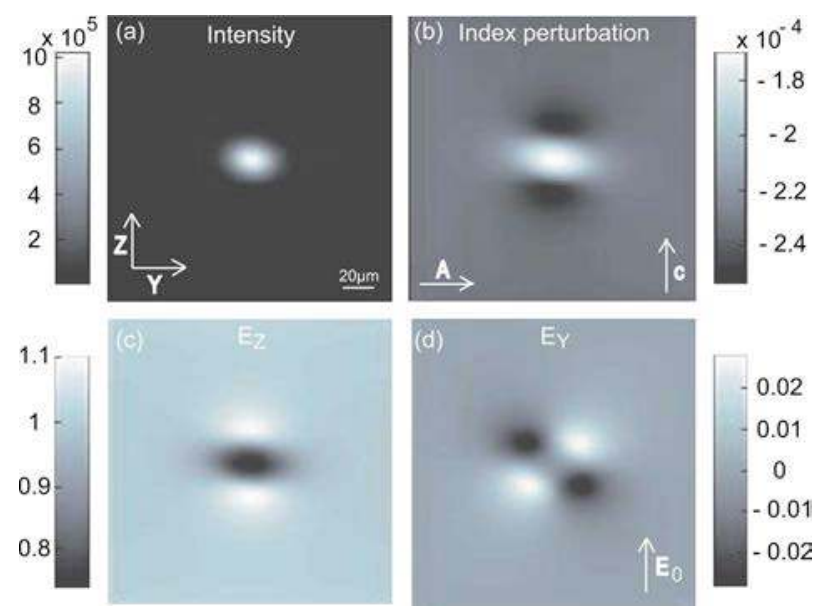

Fig. 3. (Color online) Calculated light intensity distribution for (a) ordinary polarization in a transient regime, (b) refractive index distribution, and transverse components (c) $E_{Z}$ and (d) $E_{Y}$ of the space-charge field normalized to $E_{0}$ at the exit face for propagation along the $x$ crystallographic axis in a $7 \mathrm{~mm}$ long $\mathrm{LiNbO}_{3}$ crystal. Parameters are identical to those in Fig. 1 except $\lambda$ $=632 \mathrm{~nm}$, input $\mathrm{FWHM}=16 \mu \mathrm{m}$, and $E_{p h}=-26 \mathrm{kV} / \mathrm{cm}$. 
For extraordinary polarization, no tilt is present for the elliptical shape since the index perturbation only depends on the $E_{Z}$ electric field component and the $\mathrm{r}_{33}$ electro-optic coefficient, as in Eq. (7).

\section{EXPERIMENTAL RESULTS}

In order to verify the predicted behavior, the optical setup is composed of a cw visible laser source (either at $532 \mathrm{~nm}$ or $633 \mathrm{~nm}$ ) whose beam is focused at the entrance faces of congruent undoped $\mathrm{LiNbO}_{3}$ (photonic grade) samples. Beam power and polarization can be varied, thanks to the combination of a polarizer and a half-wave plate. The beam propagates perpendicular to the $c$ axis either along a $7 \mathrm{~mm}$ or $20 \mathrm{~mm}$ long distance in the crystal, depending on the sample. A microscope objective images the exit face of the sample on a CCD camera. An external electric field of typically $E_{0}=40 \mathrm{kV} / \mathrm{cm}$ amplitude is applied in the direction of the $\mathrm{LiNbO}_{3}$ crystal $c$ axis in order to induce a self-focusing effect [10]. We note that no light beam is used to artificially increase the sample dark irradiance.

Experiments are first performed for a beam at $532 \mathrm{~nm}$ focused to a $12 \mu \mathrm{m}$ FWHM spot at the input face of a $20 \mathrm{~mm}$ long $\mathrm{LiNbO}_{3}$ sample, and propagation is along the $y$ crystallographic axis.

Dynamics of self-trapping are presented in Figs. 4 and 5 for extraordinary and ordinary polarized light, respectively, until best focusing is reached. We observe, surprisingly, that self-trapped beams are obtained for both polarizations, as revealed by the similar beam sizes present at the input and output crystal face. Indeed, slightly elliptical beams with a FWHM of $12 \mu \mathrm{m}$ and $14 \mu \mathrm{m}$, respectively, in the directions parallel $(z)$ and perpendicular $(x)$ to the applied field are formed. Note that longer induction time is, however, necessary for ordinary light.

The self-trapped beam depicted in Fig. 4(f) is in good agreement with the calculated light intensity distribution shown in Fig. 1(a). However, despite the weaker electrooptic coefficient $r_{13}$ associated with ordinary polarization, an efficiently trapped beam is observed experimentally for ordinary light. To explain this fact the polarization dependence of the photovoltaic effect has to be considered.
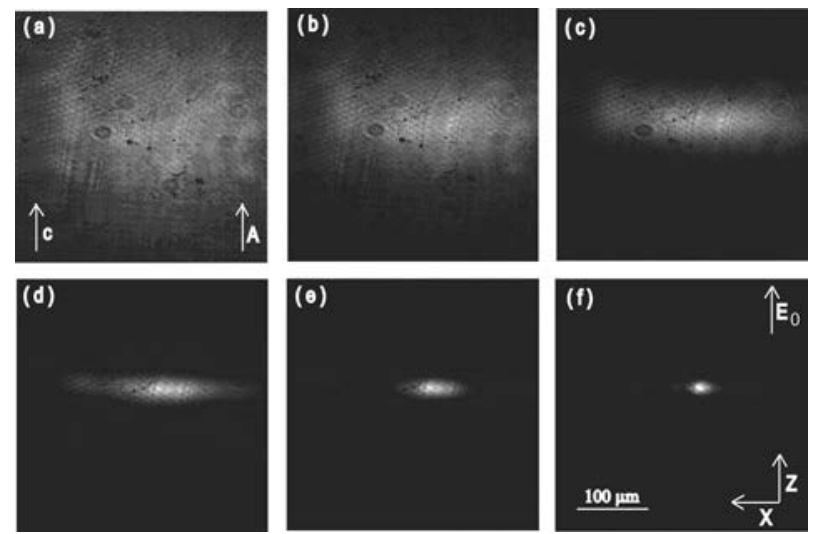

Fig. 4. Self-focusing dynamic of an extraordinary polarized beam for $E_{0}=40 \mathrm{kV} / \mathrm{cm}, \lambda=532 \mathrm{~nm}$, input power $=114 \mu \mathrm{W}$, and input beam $\mathrm{FWHM}=12 \mu \mathrm{m}$. Images are taken at regular intervals from (a) $t=0 \mathrm{~s}$ for a free diffracting beam to (f) $t=450 \mathrm{~s}$ for the most confined beam.
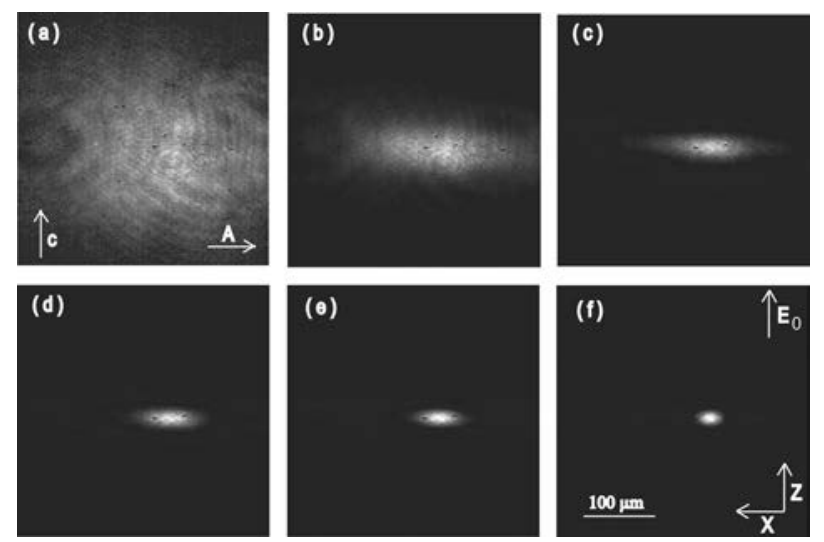

Fig. 5. Self-focusing dynamic of an ordinary polarized beam for $E_{0}=40 \mathrm{kV} / \mathrm{cm}$, input power $=114 \mu \mathrm{W}$, and input beam FWHM $=12 \mu \mathrm{m}$. Images are taken at regular intervals from (a) $\mathrm{t}=0$ for a free diffracting beam to (f) $t=1020 \mathrm{~s}$ for the most confined beam.

We thus performed additional simulations for ordinary light, considering $E_{p h}$ as a free parameter.

The best fit with the experiment is obtained for $E_{p h}$ $=-24 \mathrm{kV} / \mathrm{cm}$ (Fig. 6).

For self-trapping in $\mathrm{LiNbO}_{3}$, the photorefractive spacecharge field amplitude is given by a competition between the applied field and the adverse photovoltaic field. As a consequence, in the studied configurations the weaker photovoltaic effect linked to ordinary polarization permits the formation of a larger space-charge field that compensates for the low value of the electro-optic coefficient $r_{13}$. Similar self-induced waveguides are then formed for both polarizations. In addition, as observed experimentally, formation time is expected to be longer for ordinary than for extraordinary polarized light since a space-charge field of larger amplitude is generated for ordinary polarization. Finally, a comparison between experiment and theory allows us to deduce a ratio $\beta_{33} / \beta_{31}=1.46$ between the photovoltaic coefficients. This result is consistent with previously published values [34], $\beta_{p h 33}=4 \times 10^{-9} \mathrm{~cm} / \mathrm{V}$
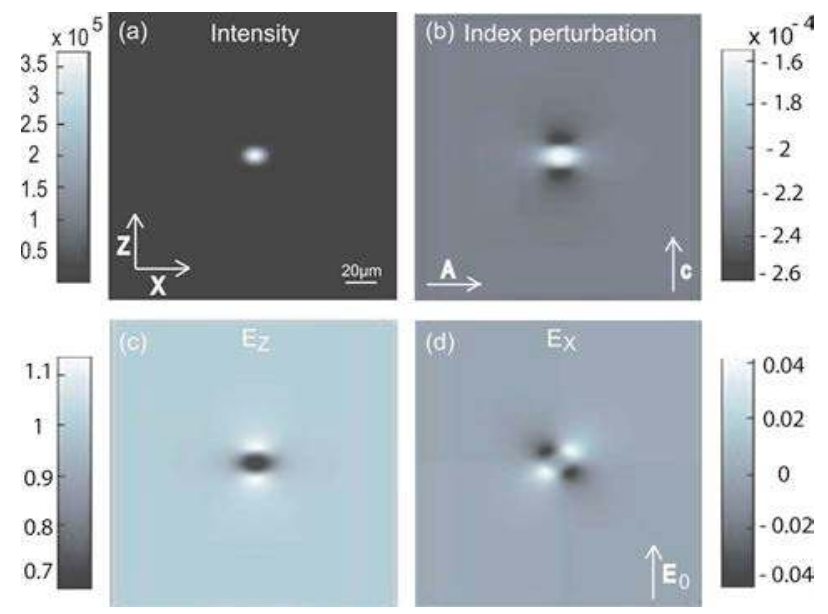

Fig. 6. (Color online) Calculated light intensity distribution for (a) ordinary polarization $\mathrm{FWHM}_{x}=14 \mu \mathrm{m}, \mathrm{FWHM}_{z}=10 \mu \mathrm{m}$, (b) refractive index distribution, and (c) transverse components $E_{Z}$ and (d) $E_{X}$ of the space-charge field normalized to $E_{0}$ at the exit face for light propagation along the $y$ crystallographic axis in a $20 \mathrm{~mm}$ long $\mathrm{LiNbO}_{3}$ crystal for $E_{p h}=-24 \mathrm{kV} / \mathrm{cm}$. 
and $\beta_{p h 31}=2 \times 10^{-9} \mathrm{~cm} / \mathrm{V}$. In a second stage we study a different electro-optic configuration. Beam propagation along the $x$ crystallographic axis in a $7 \mathrm{~mm}$ long $\mathrm{LiNbO}_{3}$ sample is now considered. When biased to $E_{0}=40 \mathrm{kV} / \mathrm{cm}$, similar results than in a previous configuration (Fig. 4) are observed, as expected from theory. However, when ordinary polarized light is launched, a different beam trapping behavior than for the $y$ propagation is observed. Indeed, the beam intensity redistributes into an elliptical shape, which is now tilted with respect to the crystal $c$ axis. This phenomenon is better observed during the initial self-trapping stage as depicted in Fig. 7.

Note that a long wavelength is used $(632 \mathrm{~nm})$ in the latter experiment, which explains the greater response time as compared with former experiments (532 nm). As predicted by the above theory, appearance of the tilted ellipse is due to the presence of two transverse space-charge field components, namely $E_{Y}$ and $E_{Z}$. Both components now combine to give the induced waveguide as specified by Eq. (9). The resulting anisotropy is, however, hardly measurable when strong focusing is reached since ellipticity becomes too weak. Such anisotropy was mentioned in a recent paper on vortex beam propagation in photorefractive $\mathrm{LiNbO}_{3}$ [35]. Note that for the reported experiments, self-trapped beams have identical dimensions for power varying from $\mu \mathrm{w}\left(I=10^{3} \mathrm{~W} / \mathrm{m}^{2}\right)$ up to $200 \mu \mathrm{w}(I$ $=10^{5} \mathrm{~W} / \mathrm{m}^{2}$ ), while increasing power reduces the formation time in accordance with the basic single deep-center model.

Finally, guiding properties of induced waveguides have been assessed in the near infrared. Self-trapped beams are first formed with $E_{0}=40 \mathrm{kV} / \mathrm{cm}$ with either ordinary or extraordinary light polarizations. Once the best trapping level is reached, the trapped beam and the applied field are switched off, then memorized waveguides are probed with near-IR beams. Typical results are presented in Fig. 8 for an extraordinary polarized probed beam. On the one hand, it shows that, when induced by an extraordinary polarized self-trapped beam in the visible range, waveguides are not strong enough to properly guide near-IR beams (fig. 8(a) and 8(b)). Losses are already detectable at $1.06 \mu \mathrm{m}$ (Fig. 8(a)) but are more obvious at $1.55 \mu \mathrm{m}$ (Fig. 8(b)). The wavelength dependence is expected from simple waveguide theory. On the other hand, when induced by ordinary polarized beams, waveguides
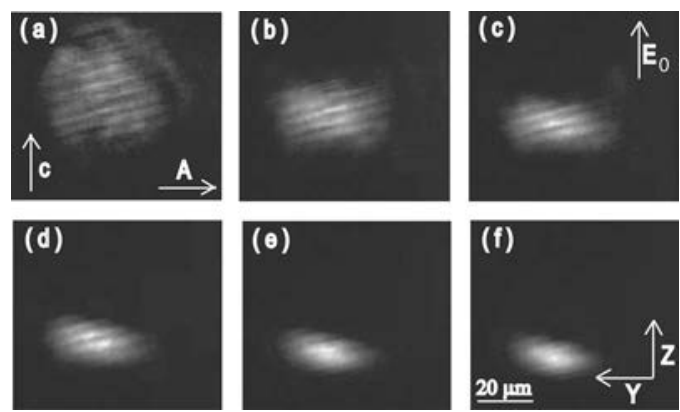

Fig. 7. Self-focusing dynamic for an ordinary polarized beam propagating along the $x$ crystallographic axis for $E_{0}=40 \mathrm{kV} / \mathrm{cm}$, $\lambda=632 \mathrm{~nm}, L=7 \mathrm{~mm}$, and $\mathrm{P}=400 \mu \mathrm{W}$. Images are taken at regular time intervals from (a) a free diffracting beam to (f) $t$ $=70 \mathrm{~min}$. Interferences are caused by CCD sensor internal reflection.
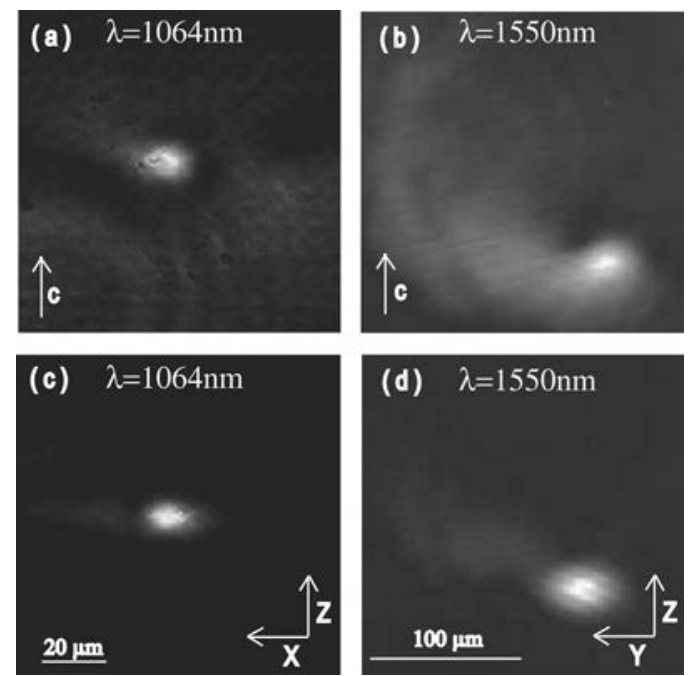

Fig. 8. Images at the exit face of waveguides formed with $(a, b)$ extraordinary and $(c, d)$ ordinary polarized trapped beam when excited with an extraordinary polarized probe at either $(\mathrm{a}, \mathrm{c})$ $1.06 \mu \mathrm{m}$ or $(\mathrm{b}, \mathrm{d}) 1.5 \mu \mathrm{m}$ wavelengths.

display much stronger guiding properties. For instance, a probe at $1.06 \mu \mathrm{m}$ is efficiently confined (Fig. 8(c)) and even a $1.55 \mu \mathrm{m}$ wavelength beam can be properly guided (Fig. 8(d)). These results clearly demonstrate that ordinary polarized spatial solitons induce deeper waveguides than extraordinary polarized solitons in $\mathrm{LiNbO}_{3}$. Indeed, a fixed waveguide created with ordinary polarization profits from a deeper space-charge field in conjunction with a larger electro-optic $r_{33}$ coefficient when probed with extraordinary polarization.

\section{CONCLUSION}

It is shown experimentally that a similar self-trapping behavior is observed in photorefractive $\mathrm{LiNbO}_{3}$ for extraordinary and for ordinary polarized beams. This polarization independence is due to a balance between electrooptic and photovoltaic coefficients, as confirmed by a timedependent $(2+1) \mathrm{D}$ photorefractive numerical model. Moreover, additional photorefractive anisotropy is revealed for a particular electro-optic configuration because both space-charge field components along and perpendicular to the $c$ axis contributes to beam self-trapping. Finally, most efficient waveguides are shown to be formed when writing beams are ordinary polarized. Such photoinduced waveguides are able to properly guide near-IF signals.

\section{REFERENCES}

1. S. Maneuf, R. Desailly, and C. Froehly, "Stable selftrapping of laser beams: observation in a nonlinear planar waveguide," Opt. Commun. 65, 193-198 (1988).

2. M. Segev, B. Crosignani, A. Yariv, and B. Fischer, "Spatial solitons in photorefractive media," Phys. Rev. Lett. 68, 923-926 (1992).

3. G. F. Calvo, B. Sturman, F. Agulló-López, and M. Carrascosa1, "Solitonlike beam propagation along lightinduced singularity of space charge in fast photorefractive media," Phys. Rev. Lett. 89, $033902-033906$ (2002). 
4. M. Asaro, M. Sheldon, Z. Chen, O. Ostroverkhova, and W. E. Moerner, "Soliton-induced waveguides in an organic photorefractive glass," Opt. Lett. 30, 519-521 (2005).

5. H. Trompeter, W. Krolikowski, D. N. Neshev, A. S. Desyatnikov, A. A. Sukhorukov, Y. S. Kivshar, T. Pertsch, U. Peschel, and F. Lederer, "Bloch oscillations and Zener tunneling in two-dimensional photonic lattices," Phys. Rev. Lett. 96, 053903-053907 (2006).

6. M. F. Shih, M. Segev, and G. Salamo, "Circular waveguides induced by two dimensional bright steady state photorefractive spatial screening solitons," Opt. Lett. 21, 931-933 (1996).

7. M. Peccianti, C. Conti, and G. Assanto, "All-optical switching and logic gating with spatial solitons in liquid crystals," Appl. Phys. Lett. 81, 3335-3337 (2002).

8. J. S. Atchison, K. Al-hemyari, C. N. Ironside, R. S. Grant, and W. Sibbett, "Observation of spatial solitons in AlGaAs waveguides," Electron. Lett. 28, 1879-1880 (1992).

9. A. Barthelemy, S. Maneuf, and C. Froehly, "Propagation soliton et auto-confinement de faisceaux laser par non linearité optique de Kerr," Opt. Commun. 55, 201-206 (1985).

10. E. Fazio, F. Renzi, R. Rinaldi, M. Bertolotti, M. Chauvet, M. Ramadan, A. Petris, and V. I. Vlad, "Screening-photovoltaic bright solitons in lithium niobate and associated singlemode waveguides," Appl. Phys. Lett. 85, 2193-2195 (2004).

11. R. Ryf, M. Wiki, G. Montemezzani, P. Gunter, and A. A. Zozulya, "Launching one-transverse-dimensional photorefractive solitons in $\mathrm{KNbO}_{3}$ crystals," Opt. Commun. 159, 339-348 (1999).

12. M. Chauvet, S. A. Hawkins, G. J. Salamo, M. Segev, D. F. Bliss, and G. Bryant, "Self-trapping of planar optical beams by use of the photorefractive effect in InP:Fe," Opt. Lett. 21, 1333-1335 (1996).

13. W. E. Torruellas, Z. Wang, D. J. Hagan, E. W. VanStryland, G. I. Stegeman, L. Torner, and C. R. Menyuk, "Observation of two-dimensional spatial solitary waves in a quadratic medium,” Phys. Rev. Lett. 74, 5037-5039 (1995).

14. G. Assanto and M. Peccianti, "Spatial solitons in nematic liquid crystals," IEEE J. Quantum Electron. 39, 13-21 (2003).

15. J. F. Henninot, J. F. Blach, and M. Warenghem, "Experimental study of the nonlocality of spatial optical solitons excited in nematic liquid crystal," J. Opt. A, Pure Appl. Opt. 9, 20-25 (2007).

16. G. Duree, J. Schultz, G. Salamo, M. Segev, A. Yariv, B. Crosignani, P. Di Porto, E. Sharp, and R. Neurgaonkar, "Observation of self-trapping of an optical beam due to the photorefractive effect," Phys. Rev. Lett. 71, 533-536 (1993).

17. D. N. Neshev, T. J. Alexander, E. A. Ostrovskaya, Y. S. Kivshar, H. Martin, I. Makasyuk, and Z. Chen, "Observation of discrete vortex solitons in optically induced photonic lattices," Phys. Rev. Lett. 92, 123903-123907 (2004).

18. U. Kang, G. I. Stegeman, J. S. Aitchison, and N. Akhmediev, "Observation of Manakov spatial solitons in AlGaAs planar waveguides," Phys. Rev. Lett. 76 , 3699-3702 (1996).
19. L. Friedrich, G. I. Stegeman, P. Millar, C. J. Hamilton, and J. S. Aitchison, "Dynamic electronically controlled angle steering of spatial solitons in AlGaAs slab waveguides," Opt. Lett. 23, 1438-1440 (1998).

20. M. Peccianti and G. Assanto, "Signal readdressing by steering of spatial solitons in bulk nematic liquid crystals," Opt. Lett. 26, 1690-1692 (2001).

21. M. Peccianti, A. De Rossi, G. Assanto, A. De Luca, C. Umeton, and I. C. Khoo, "Electrically assisted selfconfinement and waveguiding in planar nematic liquid crystal cells," Appl. Phys. Lett. 77, 7-9 (2000).

22. M. Segev, G. C. Valley, S. R. Singh, M. I. Carvalho, and D. N. Christodoulides, "Vector photorefractive spatial solitons," Opt. Lett. 20, 1764-1766 (1995).

23. S. R. Singh, M. I. Carvalho, and D. N. Christodoulides, "Vector interactions of steady-state planar solitons in biased photorefractive media," Opt. Lett. 20, 2177-2179 (1995).

24. M. Taya, M. C. Bashaw, M. M. Fejer, M. Segev, and G. C. Valley, "Observation of dark photovoltaic spatial soliton," Phys. Rev. A 52, 3095-3100 (1995).

25. G. Duree, M. Morin, G. Salamo, M. Segev, B. Grosignani, P. Di Porto, E. Sharp, and A. Yariv, "Dark photorefractive spatial solitons and photorefractive vortex solitons," Phys. Rev. Lett. 74, 1978-1981 (1995).

26. W. L. She, K. K. Lee, and W. K. Lee, "Observation of two-dimensional bright photovoltaic spatial solitons," Phys. Rev. Lett. 83, 3182-3185 (1999).

27. S. Mailis, C. Riziotis, I. T. Wellington, P. G. R. Smith, C. B. E. Gawith, and R. W. Eason, "Direct ultraviolet writing of channel waveguides in congruent lithium niobate single crystals," Opt. Lett. 28, 1433-1435 (2003).

28. V. Coda, M. Chauvet, F. Pettazi, and E. Fazio, "3-D integrated optical interconnect induced by self-focused beam," Electron. Lett. 42, 463-465 (2006).

29. F. Pettazi, V. Coda, M. Chauvet, and E. Fazio, "Frequencydoubling in self-induced waveguides in lithium niobate," Opt. Commun. 272, 238-241 (2007).

30. F. Devaux, V. Coda, M. Chauvet, and R. Passier, "New time-dependent photorefractive three-dimensional model: application to self-trapped beam with large bending," J. Opt. Soc. Am. B 25, 1081-1086 (2008).

31. A. Ciattoni and C. Palma, "Optical propagation in uniaxial crystals orthogonal to the optical axis: paraxial theory and beyond," J. Opt. Soc. Am. A 20, 2163-2171 (2003).

32. P. Yeh,Introduction to Photorefractive Nonlinear Optics (Wiley, 1993).

33. A. A. Zozulya and D. Anderson, "Propagation of an optical beam in a photorefractive medium in the presence of a photogalvanic nonlinearity or an externally applied electric field," Phys. Rev. A 51, 1520-1531 (1995).

34. B. Sturman and V. M. Fridkin, The photovoltaic and photoreceptive effects in noncentrosymmetric materials (Gordon and Breach Science Publishers, 1992).

35. R. Passier, F. Devaux, and M. Chauvet, "Impact of tensorial nature of the electro-optic effect on vortex beam propagation in photorefractive media," Opt. Express 16, 7134-7141 (2008). 